Journal of Mathematical Physics, Analysis, Geometry

2017, vol. 13, No. 4, pp. 402-413

doi:10.15407/mag13.04.402

\title{
On Properties of Root Elements in the Problem on Small Motions of Viscous Relaxing Fluid
}

\author{
D. Zakora \\ Voronezh State University \\ 1 University Sq., Voronezh 394006, Russia \\ E-mail: dmitry.zkr@gmail.com
}

Received October 20, 2015, revised May 11, 2016

\begin{abstract}
In the present work, the properties of root elements of the problem on small motions of a viscous relaxing fluid completely filling a bounded domain are studied. A multiple $p$-basis property of special system of elements is proven for the case where the system is in weightlessness. The solution of the evolution problem is expanded with respect to the corresponding system.
\end{abstract}

Key words: viscous fluid, compressible fluid, basis.

Mathematical Subject Classification 2010: 45K05, 58C40, 76R99.

\section{Introduction}

This paper is connected with [1] and deals with the problem on small motions of a viscous relaxing fluid completely filling a bounded domain. We study root elements of the corresponding spectral problem. A multiple $p$-basis property of special system of elements is proven for the case where the system is in weightlessness. The solution of an evolution problem is expanded with respect to the corresponding system.

This work was supported by the grant of the Russian Foundation for Basic Research (project no. 14-21-00066), Voronezh State University.

(c) D. Zakora, 2017 


\section{Statement of the Problem}

2.1. Statement of the boundary-value problem. Consider a container completely filled by a viscous inhomogeneous fluid. The fluid is said to fill a bounded region $\Omega \subset \mathbb{R}^{3}$. We introduce a system of coordinates $O x_{1} x_{2} x_{3}$ which is toughly connected with the container so that the origin of coordinates is in the region $\Omega$. In what follows, we suppose the hydrodynamical system to be in weightlessness.

The problem on small motions of a rotating viscous relaxing fluid is described by the following initial-boundary value problem (see $[1,2]$ ):

$$
\begin{gathered}
\frac{\partial \vec{u}(t, x)}{\partial t}-\rho_{0}^{-1}\left(\mu \Delta \vec{u}(t, x)+\left(\eta+3^{-1} \mu\right) \nabla \operatorname{div} \vec{u}(t, x)\right) \\
+a_{\infty} \rho_{0}^{-1 / 2} \nabla \rho(t, x)-\sum_{l=1}^{m} \int_{0}^{t} e^{-b_{l}(t-s)} a_{\infty} \rho_{0}^{1 / 2} k_{l} \nabla \rho(s, x) d s=\vec{f}(t, x), \\
\frac{\partial \rho(t, x)}{\partial t}+a_{\infty} \rho_{0}^{1 / 2} \operatorname{div} \vec{u}(t, x)=0 \quad(\text { in } \Omega), \quad \vec{u}(t, x)=\overrightarrow{0} \quad(\text { on } S:=\partial \Omega), \\
\vec{u}(0, x)=\vec{u}^{0}(x), \quad \rho(0, x)=\rho^{0}(x)
\end{gathered}
$$

where $\rho_{0}=$ const is the given stationary density of the fluid, $a_{\infty}$ is the given sound velocity, $\vec{f}(t, x)$ is a weak field of external forces, $\vec{u}(t, x)$ is the field of the velocities in the fluid, $a_{\infty}^{-1} \rho_{0}^{1 / 2} \rho(t, x)$ is the dynamic density of the fluid, $\mu$ and $\eta$ are the dynamic and the second viscosity of the fluid. The numbers $b_{l}^{-1}$ are used as the times of relaxation in the system $\left(0<b_{l}<b_{l+1}(l=\overline{1, m-1})\right)$, and $k_{l}>$ 0 are some structural constants.

2.2. Operator formulation of the problem. To pass to the operator formulation of the problem, we introduce basic spaces and a number of operators (see $[2])$.

Let us introduce a vector Hilbert space $\vec{L}_{2}\left(\Omega, \rho_{0}\right)$ with the scalar product and the norm

$$
(\vec{u}, \vec{v})_{\vec{L}_{2}\left(\Omega, \rho_{0}\right)}:=\int_{\Omega} \rho_{0} \vec{u}(x) \cdot \overline{\vec{v}(x)} d \Omega, \quad\|\vec{u}\|_{\vec{L}_{2}\left(\Omega, \rho_{0}\right)}^{2}=\int_{\Omega} \rho_{0}|\vec{u}(x)|^{2} d \Omega .
$$

We introduce a scalar Hilbert space $L_{2}(\Omega)$ of the square summable functions in the region $\Omega$, and also its subspace $L_{2, \Omega}:=\left\{f \in L_{2}(\Omega) \mid(f, 1)_{L_{2}(\Omega)}=0\right\}$.

Lemma 1. The following statements hold.

1. Let the boundary $S$ of the region $\Omega$ belong to the class $C^{2}$. Consider a boundary-value problem

$$
-\rho_{0}^{-1}\left(\mu \Delta \vec{u}+\left(\eta+3^{-1} \mu\right) \nabla \operatorname{div} \vec{u}\right)=\vec{w} \quad(\text { in } \Omega), \quad \vec{u}=\overrightarrow{0} \quad(\text { on } S) .
$$


For every field $\vec{w} \in \vec{L}_{2}\left(\Omega, \rho_{0}\right)$, the generalized solution of the problem given by the formula $\vec{u}=A^{-1} \vec{w}$ exists and it is unique. The operator $A$ is selfadjoint and positive definite in $\vec{L}_{2}\left(\Omega, \rho_{0}\right)$. The operator $A^{-1}$ belongs to the class $\mathfrak{S}_{p}$ $(p>3 / 2)$. In addition, $\mathscr{D}\left(A^{1 / 2}\right)=\left\{\vec{u} \in \vec{W}_{2}^{1}(\Omega) \mid \vec{u}=\overrightarrow{0}(\right.$ on $\left.S)\right\}$.

2. Let us define an operator $B \vec{u}:=a_{\infty} \rho_{0}^{1 / 2} \operatorname{div} \vec{u}, \mathscr{D}(B):=\mathscr{D}\left(A^{1 / 2}\right)$. The adjoint operator $B^{*} \rho=-a_{\infty} \rho_{0}^{-1 / 2} \nabla \rho, \mathscr{D}\left(B^{*}\right)=W_{2}^{1}(\Omega) \cap L_{2, \Omega}, B^{*} B A^{-1} \in$ $\mathscr{L}\left(\vec{L}_{2}\left(\Omega, \rho_{0}\right)\right)$.

3. The operator $Q:=B A^{-1 / 2}$ is bounded: $Q \in \mathscr{L}\left(\vec{L}_{2}\left(\Omega, \rho_{0}\right), L_{2, \Omega}\right)$. The operator $Q^{+}:=A^{-1 / 2} B^{*}$ admits extension to the bounded operator $Q^{*}: \overline{Q^{+}}=Q^{*}$.

Using the operators introduced above, we can write problem (1) as a system of two equations with initial-value conditions in a Hilbert space $H_{0}:=\vec{L}_{2}\left(\Omega, \rho_{0}\right) \oplus$ $L_{2, \Omega}$ :

$$
\left\{\begin{array}{l}
\frac{d \vec{u}}{d t}+2 \omega_{0} i S \vec{u}+A \vec{u}-B^{*} \rho+\sum_{l=1}^{m} \int_{0}^{t} e^{-b_{l}(t-s)} B^{*} \rho_{0} k_{l} \rho(s) d s=\vec{f}(t), \\
\frac{d \rho}{d t}+B \vec{u}=0, \quad(\vec{u}(0) ; \rho(0))^{\tau}=\left(\vec{u}^{0} ; \rho^{0}\right)^{\tau} .
\end{array}\right.
$$

The upper index $\tau$ denotes the transposition.

Definition 1. A strong solution to problem (2) is said to be a strong solution to initial-value problem (1). A function $\zeta(t):=(\vec{u}(t) ; \rho(t))^{\tau}$ is a strong solution to problem (2) if $\zeta(t) \in \mathscr{D}(A) \oplus \mathscr{D}\left(B^{*}\right)$ for all $t \in \mathbb{R}_{+},\left(A \vec{u}(t) ; B^{*} \rho(t)\right)^{\tau} \in C\left(\mathbb{R}_{+}, H_{0}\right)$, $(\vec{u}(t) ; \rho(t))^{\tau} \in C^{1}\left(\mathbb{R}_{+}, H_{0}\right)$, and $\zeta(t)$ satisfies (2) for all $t \in \mathbb{R}_{+}:=[0,+\infty)$.

2.3. Reduction to the first-order differential equation. Let us suppose that problem (2) has a strong solution $\vec{u}(t), \rho(t)$ and $\rho^{0} \in \mathscr{D}\left(B^{*}\right)$. From Lemma 1 , it follows that $\vec{u}(t)$ and $\rho(t)$ satisfy the system

$$
\left\{\begin{aligned}
\frac{d \vec{u}}{d t}+ & A^{1 / 2}\left\{A^{1 / 2} \vec{u}-Q^{*}\left[1-\sum_{l=1}^{m} \frac{\rho_{0} k_{l}}{b_{l}}\right] \rho\right. \\
& \left.\quad-\sum_{l=1}^{m} Q^{*} \int_{0}^{t} e^{-b_{l}(t-s)} \frac{\rho_{0} k_{l}}{b_{l}} \frac{d \rho(s)}{d s} d s\right\}=\vec{f}(t)+\sum_{l=1}^{m} e^{-b_{l} t} \frac{\rho_{0} k_{l}}{b_{l}} B^{*} \rho^{0}, \\
\frac{d \rho}{d t}+ & Q A^{1 / 2} \vec{u}=0, \quad(\vec{u}(0) ; \rho(0))^{\tau}=\left(\vec{u}^{0} ; \rho^{0}\right)^{\tau} .
\end{aligned}\right.
$$

In what follows, we suppose the physical parameters satisfy the condition

$$
\varphi_{0}:=1-\sum_{l=1}^{m} \frac{\rho_{0} k_{l}}{b_{l}}>0 .
$$


This condition supposes that the times of relaxation $b_{l}^{-1}$ and the structural constants $k_{l}(l=\overline{1, m})$ are sufficiently small.

Let us define the following objects connected with the function $\rho(t)$ :

$$
\varphi_{0}^{1 / 2} \rho(t)=: r(t), \quad \int_{0}^{t} e^{-b_{l}(t-s)}\left[\frac{\rho_{0} k_{l}}{b_{l}}\right]^{1 / 2} \frac{d \rho(s)}{d s} d s=: r_{l}(t) \quad(l=\overline{1, m}) .
$$

The functions $r(t)$ and $r_{l}(t)(l=\overline{1, m})$ are continuously differentiable. From (3), it follows that these functions satisfy the system

$$
\left\{\begin{array}{l}
\frac{d \vec{u}}{d t}+A^{1 / 2}\left\{A^{1 / 2} \vec{u}-\varphi_{0}^{1 / 2} Q^{*} r-\sum_{l=1}^{m}\left[\frac{\rho_{0} k_{l}}{b_{l}}\right]^{1 / 2} Q^{*} r_{l}\right\}=\vec{f}_{0}(t), \\
\frac{d r}{d t}+\varphi_{0}^{1 / 2} Q A^{1 / 2} \vec{u}=0, \frac{d r_{l}}{d t}+\left[\frac{\rho_{0} k_{l}}{b_{l}}\right]^{1 / 2} Q A^{1 / 2} \vec{u}+b_{l} r_{l}=0 \quad(l=\overline{1, m}), \\
\vec{f}_{0}(t):=\vec{f}(t)+\sum_{l=1}^{m} e^{-b_{l} t} \frac{\rho_{0} k_{l}}{b_{l}} B^{*} \rho^{0} .
\end{array}\right.
$$

Let us write system (6) as a first-order differential equation in a Hilbert space $\mathscr{H}:=H \oplus \mathscr{H}_{0}\left(H:=\vec{L}_{2}\left(\Omega, \rho_{0}\right), \mathscr{H}_{0}:=\oplus_{l=1}^{m+1} L_{2, \Omega}\right):$

$$
\frac{d \xi}{d t}+\mathscr{A} \xi=\mathscr{F}(t), \quad \xi(0)=\xi^{0},
$$

where

$$
\begin{gathered}
\xi:=(\vec{u} ; w)^{\tau}, \quad w:=\left(r ; r_{1} ; \ldots ; r_{m}\right)^{\tau}, \quad \xi^{0}:=\left(\vec{u}^{0} ; w^{0}\right)^{\tau}, \\
w^{0}:=\left(\varphi_{0}^{1 / 2} \rho^{0} ; 0 ; \ldots ; 0\right)^{\tau}, \quad \mathscr{F}(t):=\left(\overrightarrow{f_{0}}(t) ; 0 ; \ldots ; 0\right)^{\tau} .
\end{gathered}
$$

The operator $\mathscr{A}$ satisfies the following formulae:

$$
\begin{gathered}
\mathscr{A}=\operatorname{diag}\left(A^{1 / 2}, \mathscr{I}_{0}\right)\left(\begin{array}{cc}
I & \mathbb{Q}^{*} \\
-\mathbb{Q} & \mathscr{G}
\end{array}\right) \operatorname{diag}\left(A^{1 / 2}, \mathscr{I}_{0}\right), \\
\mathscr{A}=\left(\begin{array}{cc}
I & 0 \\
-\mathbb{Q} A^{-1 / 2} & \mathscr{J}_{0}
\end{array}\right) \operatorname{diag}\left(A, \mathscr{G}+\mathbb{Q}^{*}\right)\left(\begin{array}{cc}
I & A^{-1 / 2} \mathbb{Q}^{*} \\
0 & \mathscr{J}_{0}
\end{array}\right), \\
\mathscr{D}(\mathscr{A})=\left\{\xi=(\vec{u} ; w)^{\tau} \in \mathscr{H} \mid \vec{u}+A^{-1 / 2} \mathbb{Q}^{*} w \in \mathscr{D}(A)\right\},
\end{gathered}
$$

where $I$ and $\mathscr{J}_{0}$ are the identity operators in $H$ and $\mathscr{H}_{0}$, respectively,

$$
\begin{gathered}
\mathcal{Q}:=\left(-\varphi_{0}^{1 / 2} Q,-\left[\frac{\rho_{0} k_{1}}{b_{1}}\right]^{1 / 2} Q, \ldots,-\left[\frac{\rho_{0} k_{1}}{b_{m}}\right]^{1 / 2} Q\right)^{\tau}, \\
\mathscr{G}:=\operatorname{diag}\left(0, b_{1} I, \ldots, b_{m} I\right) .
\end{gathered}
$$

From [1], it follows that $\mathscr{A}$ is a maximal sectorial (and accretive) operator. From this and (7) one can obtain the theorem on strong solvability of problem (1). 
Theorem 1 (see [1]). Let the field $\vec{f}(t, x)$ satisfy the Hölder condition $\forall \tau \in$ $\mathbb{R}_{+} \exists K=K(\tau)>0, k(\tau) \in(0,1]:\|\vec{f}(t)-\vec{f}(s)\|_{\vec{L}_{2}\left(\Omega, \rho_{0}\right)} \leq K|t-s|^{k}$ for all $0 \leq$ $s, t \leq \tau$. Then for any $\vec{u}^{0} \in \mathscr{D}(A)$ and $\rho^{0} \in \mathscr{D}\left(B^{*}\right)$ there exists a unique strong solution to initial-boundary value problem (1).

2.4. The basic spectral problems and the theorem on recalculation of root elements. We consider the spectral problem to the evolution problem $(7)$. Assuming $\mathscr{F}(t) \equiv 0$, a dependence on time for the unknown function be of the form $\xi(t)=\exp (-\lambda t) \xi$, where $\lambda$ is a spectral parameter and $\xi$ is an amplitude element, we have

$$
\mathscr{A} \xi=\lambda \xi, \quad \xi \in \mathscr{D}(\mathscr{A}) \subset \mathscr{H} .
$$

Let $\xi=(\vec{u} ; w)^{\tau} \in \mathscr{D}(\mathscr{A})$. Changing the sought element in problem (13), $\operatorname{diag}\left(A^{1 / 2}, \mathscr{I}_{0}\right) \xi=\zeta=:(\vec{v} ; w)^{\tau}$, using factorization (10), we have the following spectral problem:

$$
\mathscr{A}(\lambda) \zeta:=\left(\begin{array}{cc}
I-\lambda A^{-1} & \mathcal{Q}^{*} \\
-Q & \mathscr{Q}-\lambda
\end{array}\right)\left(\begin{array}{c}
\vec{v} \\
w
\end{array}\right)=0, \quad \zeta \in \mathscr{H}=H \oplus \mathscr{H}_{0} .
$$

Let $\lambda \notin\left\{0, b_{1}, \ldots, b_{m}\right\}=\sigma(\mathscr{G})$. From (14) it follows that

$$
L(\lambda) \vec{v}:=\left[I-\lambda A^{-1}+Q^{*}(\mathscr{G}-\lambda)^{-1} Q\right] \vec{v}=0, \quad \vec{v} \in H .
$$

From [1], we obtain the theorem on the spectrum of the operator $\mathcal{A}$.

Theorem 2 (see $[1$, Theorems 2, 4]). The following statements hold.

1. $\sigma_{\mathrm{ess}}(\mathscr{A})=\Lambda_{E} \cup \Lambda_{L}$, where

$$
\begin{aligned}
& \Lambda_{E}:=\left\{\lambda \in \mathbb{C} \mid 1-\sum_{l=1}^{m} \frac{\rho_{0} k_{l}}{b_{l}-\lambda}=\lambda \frac{4 \mu+3 \eta}{3 a_{\infty}^{2} \rho_{0}}\right\}, \\
& \Lambda_{L}:=\left\{\lambda \in \mathbb{C} \mid 1-\sum_{l=1}^{m} \frac{\rho_{0} k_{l}}{b_{l}-\lambda}=\lambda \frac{7 \mu+3 \eta}{3 a_{\infty}^{2} \rho_{0}}\right\} .
\end{aligned}
$$

The set $\mathbb{C} \backslash \sigma_{\text {ess }}(\mathscr{A})$ consists of regular points and isolated eigenvalues of finite multiplicity of the operator $\mathcal{A}$.

2. $\lambda=0$ is not an eigenvalue of the operator A. If $\left\|A^{-1 / 2}\right\|<b_{q}^{-1 / 2}$, then $\lambda=$ $b_{q}$ is not an eigenvalue of the operator $\mathcal{A}$. Otherwise the point $\lambda=b_{q}$ can be an eigenvalue of finite multiplicity of the operator $\mathcal{A}$. 
3. If $\lambda_{0}$ is a non-real eigenvalue of the operator $\mathcal{A}$, then

$$
\begin{gathered}
\gamma_{1}:=\left[2\left\|A^{-1 / 2}\right\|^{2}\right]^{-1}<\operatorname{Re} \lambda_{0}<b_{m}+\|Q\|^{2}+\|Q\|\left(b_{m}+\|Q\|^{2}\right)^{1 / 2}=: \gamma_{2}, \\
\left|\lambda_{0}\right|^{2}<\left(b_{m}+2\|Q\|^{2}+2\|Q\|\left(b_{m}+\|Q\|^{2}\right)^{1 / 2}\right)\left(2 b_{m}+\|Q\|^{2}\right) .
\end{gathered}
$$

The spectrum of the operator $\mathscr{A}$ is real if the following condition holds:

$$
2\left\|A^{-1 / 2}\right\|^{2} \leqslant\left(b_{m}+\|Q\|^{2}+\|Q\|\left(b_{m}+\|Q\|^{2}\right)^{1 / 2}\right)^{-1} .
$$

Let us introduce the following definition.

Definition 2 (see [3, p. 61]). Let $\lambda_{0}$ and $\vec{v}_{0}$ be an eigenvalue and an eigenvector of the operator pencil $L(\lambda)$, i.e., $L\left(\lambda_{0}\right) \vec{v}_{0}=0$. The elements $\vec{v}_{1}, \vec{v}_{2}, \ldots, \vec{v}_{n-1}$ are said to be adjoint to $\vec{v}_{0}$ if $\sum_{k=0}^{j}(k !)^{-1} L^{(k)}\left(\lambda_{0}\right) \vec{v}_{j-k}=0(j=1,2, \ldots, n-1)$. The number $n$ is called the length of the chain $\vec{v}_{0}, \vec{v}_{1}, \ldots, \vec{v}_{n-1}$ of root elements.

From [4], we obtain the following theorem on connection between root elements of the operator $\mathscr{A}$ and the operator pencil $L(\lambda)$.

Theorem 3. Let $\left\{\xi_{k}=\left(\vec{u}_{k} ; w_{k}\right)^{\tau}\right\}_{k=0}^{n-1}$ denote a chain of root elements of the operator $\mathscr{A}$ and this chain corresponds to $\lambda_{0}\left(\lambda_{0} \neq 0, b_{1}, \ldots, b_{m}\right)$. Then $\left\{\vec{v}_{k}\right\}_{k=0}^{n-1}:=\left\{A^{1 / 2} \vec{u}_{k}\right\}_{k=0}^{n-1}$ is a chain of root elements of the operator pencil $L(\lambda)$ and this chain corresponds to $\lambda_{0}$.

Let $\left\{\vec{v}_{k}\right\}_{k=0}^{n-1}$ denote a chain of root elements of the operator pencil $L(\lambda)$ and this chain corresponds to $\lambda_{0}$. Then $\left\{\xi_{k}=\left(A^{-1 / 2} \vec{v}_{k} ; w_{k}\right)^{\tau}\right\}_{k=0}^{n-1}$, where $w_{k}:=$ $\sum_{l=0}^{k}\left(\mathscr{G}-\lambda_{0}\right)^{-(k-l+1)} \mathbb{Q} \vec{v}_{l}$, is a chain of root elements of the operator $\mathscr{A}$.

\section{A Multiple $p$-Basicity of Special System of Elements}

3.1. The theorem on completeness and $p$-basicity of root elements of the operator $\mathcal{A}$. The following considerations are based on the theory of spaces with indefinite metrics (see $[5,6]$ ). Therefore we assume that $\mathscr{H}=\mathscr{H}_{+} \oplus$ $\mathscr{H}_{-}$, where $\mathscr{H}_{+}:=H=\vec{L}_{2}\left(\Omega, \rho_{0}\right), \mathscr{H}_{-}:=\mathscr{H}_{0}$. We recall here some concepts and facts of this theory.

Define $\mathscr{F}:=\operatorname{diag}\left(I,-\mathscr{I}_{0}\right)$ and introduce in $\mathscr{H}$ an indefinite scalar product by the formula $\left[\xi_{1}, \xi_{2}\right]:=\left(\mathscr{F} \zeta_{1}, \zeta_{2}\right) \mathscr{H}=\left(\vec{v}_{1}, \vec{v}_{2}\right) \mathscr{H}_{+}-\left(w_{1}, w_{2}\right) \mathscr{H}_{-}$. Denote by $\mathscr{P}_{+}$

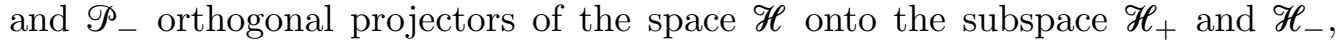
respectively: $\mathscr{P}_{+} \mathscr{H}=\mathscr{H}_{+}, \mathscr{P}_{-} \mathscr{H}=\mathscr{H}_{-}$.

A subspace $L_{+}$is called nonnegative if $[\xi, \xi] \geqslant 0$ for all $\xi \in L_{+}$, and maximal nonnegative $\left(L_{+} \in \mathfrak{M}^{+}\right)$if it is not a principle part of any other nonnegative subspace. In the same way, we can define nonpositive subspace $L_{-}$. 
From [5, p. 70]), it follows that $L_{+} \in \mathfrak{M}^{+}$if there exists a restriction $K_{+}$: $\mathscr{H}_{+} \rightarrow \mathscr{H}_{-}\left(\left\|K_{+}\right\| \leqslant 1\right)$ such that $L_{+}=\left\{\xi=\xi_{+}+K_{+} \xi_{+}: \xi_{+} \in \mathscr{H}_{+}\right\}$. This restriction is called an angular operator of the subspace $L_{+}$.

A positive subspace $L_{+}$is said to be uniformly positive if it is a Hilbert space with respect to the scalar product generated by the indefinite metric.

We say that the subspace $L_{+}$belongs to the class $h^{+}$if it can be represented as the $\mathscr{F}$-orthogonal sum of the uniformly positive subspace and the finitedimensional neutral subspace. In particular, $L_{+} \in h^{+}$if $K_{+} \in \mathfrak{S}_{\infty}$ (see [5, p. 84]).

Let $L_{ \pm} \in \mathfrak{M}^{ \pm}$. If $L_{+}$and $L_{-}$are $\mathscr{g}$-orthogonal, then we say that $L_{+}$and $L_{-}$ form a dual pair $\left\{L_{+}, L_{-}\right\}$. We can write $\left\{L_{+}, L_{-}\right\} \in h$, if $L_{ \pm} \in h^{ \pm}$.

We say that a continuous $\mathscr{F}$-selfadjoint operator $\mathscr{B}$ belongs to Helton's class $(\mathscr{B} \in(H))$ if there exists at least one dual pair $\left\{L_{+}, L_{-}\right\} \in h$ of invariant with respect to $\mathscr{B}$-subspaces and every $\mathscr{B}$-invariant dual pair belongs to the class $h$.

Theorem 4. $\mathscr{A}^{-1} \in(H)$.

Proof. The Schur-Frobenius factorization (11) of the operator $\mathscr{A}$ and Theorem $2(0 \in \rho(\mathscr{A}))$ imply

$$
\begin{aligned}
& \mathscr{A}^{-1}=\left(\begin{array}{cc}
I & -A^{-1 / 2} \mathbb{Q}^{*} \\
0 & \mathscr{I}_{0}
\end{array}\right) \operatorname{diag}\left(A^{-1},\left(\mathscr{G}+Q_{Q} \mathbb{Q}^{*}\right)^{-1}\right)\left(\begin{array}{cc}
I & 0 \\
\mathcal{Q} A^{-1 / 2} & \mathscr{I}_{0}
\end{array}\right)
\end{aligned}
$$

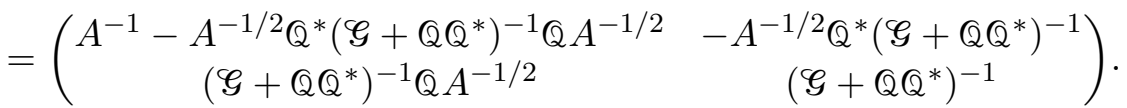

The operator $\mathscr{A}^{-1}$ is $\mathscr{F}$-selfadjoint and bounded. The compactness of the operator $A^{-1 / 2}$ implies the compactness of the operator $\mathscr{P}_{+} \mathscr{A}^{-1} \mathscr{P}_{-}$. From this and $\left[5\right.$, p. 287], it follows that the operator $\mathscr{A}^{-1}$ has a dual invariant pair $\left\{L_{+}\left(\mathscr{A}^{-1}\right), L_{-}\left(\mathscr{A}^{-1}\right)\right\}$. Let $K_{+}$denote the angular operator of invariant nonnegative subspace $L_{+}\left(\mathscr{A}^{-1}\right)$. Then $K_{+}: \mathscr{H}_{+} \rightarrow \mathscr{H}_{-},\left\|K_{+}\right\| \leqslant 1$, and

$$
L_{+}\left(\mathscr{A}^{-1}\right)=\left\{(\vec{u} ; w)^{\tau} \in \mathscr{H}_{+} \oplus \mathscr{H}_{-} \mid(\vec{u} ; w)^{\tau}=\left(\vec{u} ; K_{+} \vec{u}\right)^{\tau}, \quad \vec{u} \in \mathscr{H}_{+}\right\} .
$$

Let $\left(\vec{u}_{1} ; w_{1}\right)^{\tau}=\left(\vec{u}_{1} ; K_{+} \vec{u}_{1}\right)^{\tau} \in L_{+}\left(\mathscr{A}^{-1}\right)$. Then $\mathscr{A}^{-1}\left(\vec{u}_{1} ; K_{+} \vec{u}_{1}\right)^{\tau}=\left(\vec{u}_{2} ; K_{+} \vec{u}_{2}\right)^{\tau}$. From this and (17), we deduce the equation for angular operator $K_{+}$:

$$
\begin{aligned}
\left(\mathscr{G}+\mathbb{Q}^{*}\right)^{-1} K_{+}= & -\left(\mathscr{G}+Q_{Q^{*}}\right)^{-1} Q_{Q} A^{-1 / 2} \\
& +K_{+}\left(A^{-1}-A^{-1 / 2} \mathbb{Q}^{*}\left(\mathscr{G}+\mathbb{Q} \mathbb{Q}^{*}\right)^{-1} \mathbb{Q} A^{-1 / 2}\right) \\
& -K_{+} A^{-1 / 2} \mathbb{Q}^{*}\left(\mathscr{G}+Q_{Q^{*}}\right)^{-1} K_{+} .
\end{aligned}
$$

From $A^{-1 / 2} \in \mathfrak{S}_{\infty}$, it follows that $K_{+} \in \mathfrak{S}_{\infty}\left(\mathscr{H}_{+}\right)$. 
Remark 1 . Theorem 4 is valid for the case where the system is in the gravitational field. In particular, Theorem 4 implies that the nonreal spectrum of the operator $\mathscr{A}$ consists of at most finite number of eigenvalues with regard for their algebraic multiplicity (see [5, p. 245, Corollary 5.21]).

A system $\left\{\xi_{k}\right\}_{k=1}^{\infty}$ is said to be a Riesz basis in a space $\mathscr{H}$ if $\xi_{k}=\mathscr{T} \zeta_{k}$, where $\mathscr{T}, \mathscr{T}^{-1} \in \mathscr{L}(\mathscr{H})$, and $\left\{\zeta_{k}\right\}_{k=1}^{\infty}$ is an orthonormal basis in the space $\mathscr{H}$. If $\mathscr{T}=$ $\mathscr{I}+\mathscr{K}$, where $\mathscr{K} \in \mathfrak{S}_{p}(\mathscr{H})$, then the system $\left\{\xi_{k}\right\}_{k=1}^{\infty}$ is called a $p$-basis in the space $\mathscr{H}$.

A basis in the $\mathscr{F}$-space $\mathscr{H}$ is said to be almost $\mathscr{F}$-orthonormal if it can be represented as a unity of a finite number of elements and a set of $\mathscr{F}$-orthonormal elements, and the sets are $\mathscr{F}$-orthogonal to each other.

Let $\mathfrak{L}_{\lambda}(\mathscr{A})$ denote a root subspace of the operator $\mathscr{A}$ which corresponds to the eigenvalue $\lambda\left(\lambda \in \sigma_{p}(\mathscr{A})\right)$. Let us also introduce the following notations:

$$
\mathfrak{F}(\mathscr{A}):=\overline{\operatorname{sp}\left\{\mathfrak{L}_{\lambda}(\mathscr{A}) \mid \lambda \in \sigma_{p}(\mathscr{A})\right\}}, \quad \mathfrak{F}_{0}(\mathscr{A}):=\overline{\operatorname{sp}\left\{\operatorname{Ker}(\mathscr{A}-\lambda) \mid \lambda \in \sigma_{p}(\mathscr{A})\right\}} .
$$

We write $\lambda \in s(\mathscr{A}) \subset \mathbb{R}$ if $\operatorname{Ker}(\mathscr{A}-\lambda)$ is degenerate, i.e., there exists $\xi_{0} \in \operatorname{Ker}(\mathscr{A}-$ $\lambda)$ such that $\left[\xi_{0}, \xi\right]=0$ for all $\xi \in \operatorname{Ker}(\mathscr{A}-\lambda)$.

Theorem of T.Ya. Azizov (see [5, p. 271, Theorem 2.12]) implies the following statement.

Theorem 5. The following statements hold.

1. $\operatorname{codim} \mathfrak{F}(\mathscr{A}) \leqslant \operatorname{codim} \mathfrak{F}_{0}(\mathscr{A})<\infty$.

2. $\mathfrak{F}(\mathscr{A})=\mathscr{H} \Longleftrightarrow \operatorname{sp}\left\{\mathfrak{L}_{\lambda}(\mathscr{A}) \mid \lambda \in \sigma_{\text {ess }}(\mathscr{A}) \cap\left(\gamma_{1}, \gamma_{2}\right)\right\}$ is a non-degenerate subspace. The numbers $\gamma_{1}$ and $\gamma_{2}$ are defined in Theorem 2.

3. $\mathfrak{F}_{0}(\mathscr{A})=\mathscr{H} \Longleftrightarrow \mathfrak{L}_{\lambda}(\mathscr{A})=\operatorname{Ker}(\mathscr{A}-\lambda)$ as $\lambda \neq \bar{\lambda}$ and $s(\mathscr{A})=\emptyset$. If $\gamma_{2} \leqslant \gamma_{1}$ then $\mathfrak{F}_{0}(\mathscr{A})=\mathscr{H}$.

4. If $\mathfrak{F}_{0}(\mathscr{A})=\mathscr{H}(\mathfrak{F}(\mathscr{A})=\mathscr{H})$, then the eigenelements (root elements) of the

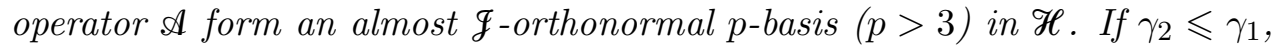
then the eigenelements of the operator $\mathscr{A}$ form a $\mathscr{F}$-orthonormal basis in $\mathscr{H}$.

Proof. Theorem 4 implies $\mathscr{A}^{-1} \in(H)$. From (18) and $A^{-1} \in \mathfrak{S}_{p}(p>3 / 2)$ it follows that $K_{+} \in \mathfrak{S}_{p}(p>3)$. From Theorem 2 we conclude that the spectrum of the operator $\mathscr{A}^{-1}$ has at most finite number of accumulation points. Therefore the operator $\mathscr{A}^{-1}$ satisfies all assumptions of T.Ya. Azizov's theorem.

1. From $\mathfrak{F}(\mathscr{A})=\mathfrak{F}\left(\mathscr{A}^{-1}\right), \mathfrak{F}_{0}(\mathscr{A})=\mathfrak{F}_{0}\left(\mathscr{A}^{-1}\right)$ we obtain the first statement.

2. $\mathfrak{F}\left(\mathscr{A}^{-1}\right)=\mathscr{H} \Longleftrightarrow \operatorname{sp}\left\{\mathfrak{L}_{\lambda^{-1}}\left(\mathscr{A}^{-1}\right) \mid \lambda^{-1} \in s\left(\mathscr{A}^{-1}\right)\right\}$ is a non-degenerate subspace. To prove $\mathfrak{F}\left(\mathscr{A}^{-1}\right)=\mathscr{H}$, we should check that $\mathfrak{L}_{\lambda^{-1}}\left(\mathscr{A}^{-1}\right)$ is a nondegenerate subspace only for those $\lambda^{-1} \in s\left(\mathscr{A}^{-1}\right)$ which are accumulation points 
of the operator $\mathscr{A}^{-1}$ (see [5, p. 271, Remark 3.8]). From $\mathfrak{L}_{\lambda^{-1}}\left(\mathscr{A}^{-1}\right)=\mathfrak{L}_{\lambda}(\mathscr{A})$ we have the following conclusion. To prove $\mathfrak{F}\left(\mathscr{A}^{-1}\right)=\mathscr{H}$, we should check that whether $\mathfrak{L}_{\lambda}(\mathscr{A})$ is a non-degenerate subspace for $\lambda \in \sigma_{\text {ess }}(\mathscr{A}) \cap s(\mathscr{A})$.

Now we should determine where the set $s(\mathscr{A})$ is positioned. Let $\lambda_{0}=\bar{\lambda}_{0} \in$ $\sigma_{p}(\mathscr{A}), \lambda_{0} \notin \sigma(\mathscr{G})$, and $\operatorname{Ker}\left(\mathscr{A}-\lambda_{0}\right)$ be degenerated. By Theorem 3, it is equivalent to the following. There exists $\vec{v}_{0} \in \operatorname{Ker} L\left(\lambda_{0}\right)$ such that $\xi_{0}=\left(A^{-1 / 2} \vec{v}_{0} ;(\mathscr{G}-\right.$ $\left.\lambda_{0}\right)^{-1}\left(\mathbb{Q} \vec{v}_{0}\right)^{\tau}$ is $\mathscr{g}$-orthogonal to all elements $\xi=\left(A^{-1 / 2} \vec{v} ;\left(\mathscr{G}-\lambda_{0}\right)^{-1} \mathbb{Q} \vec{v}\right)^{\tau}$, where $\vec{v} \in \operatorname{Ker} L\left(\lambda_{0}\right)$, i.e., $\left[\xi_{0}, \xi\right]=0$. From the above it follows that $\left(L^{\prime}\left(\lambda_{0}\right) \vec{v}_{0}, \vec{v}\right)_{H}=$ 0 . In particular, we have two equations: $\left(L\left(\lambda_{0}\right) \vec{v}_{0}, \vec{v}_{0}\right)_{H}=0,\left(L^{\prime}\left(\lambda_{0}\right) \vec{v}_{0}, \vec{v}_{0}\right)_{H}=0$. From this it follows that $\lambda_{0}$ is a multiple root of the equation

$$
\begin{gathered}
1-\lambda p-\frac{1}{\lambda}\left(q-\sum_{l=1}^{m} \frac{q_{l}}{b_{l}-\lambda}\right)=0, \\
p:=\frac{\left\|A^{-1 / 2} \vec{v}_{0}\right\|^{2}}{\left\|\vec{v}_{0}\right\|^{2}}, \quad q:=\frac{\left\|Q \vec{v}_{0}\right\|^{2}}{\left\|\vec{v}_{0}\right\|^{2}}, \quad q_{l}:=\rho_{0} k_{l} q \quad(l=\overline{1, m}) .
\end{gathered}
$$

Let us write (19) in the form

$$
\begin{aligned}
0 & =\left(\lambda-\lambda^{2} p-q\right) \prod_{l=1}^{m}\left(b_{l}-\lambda\right)+\sum_{l=1}^{m} q_{l} \prod_{k \neq l}^{m}\left(b_{k}-\lambda\right)=-p(-1)^{m} \lambda^{m+2} \\
& +(-1)^{m} \lambda^{m+1}\left[1+p \sum_{l=1}^{m} b_{l}\right]-(-1)^{m} \lambda^{m}\left[q+\sum_{l=1}^{m} b_{l}+p \sum_{i<j} b_{i} b_{j}\right]+\cdots
\end{aligned}
$$

Equation (19) has $m$ real roots $\lambda_{l}\left(\lambda_{l} \in\left(b_{l-1}, b_{l}\right), l=\overline{1, m}, b_{0}:=0\right)$ and a real double root $\lambda_{0}$. Then

$$
\begin{array}{r}
0=-p \prod_{l=1}^{m}\left(\lambda_{l}-\lambda\right)\left(\lambda-\lambda_{0}\right)^{2}=-p(-1)^{m} \lambda^{m+2}+(-1)^{m} \lambda^{m+1} p\left[2 \lambda_{0}+\sum_{l=1}^{m} \lambda_{l}\right] \\
-(-1)^{m} \lambda^{m} p\left[\lambda_{0}^{2}+2 \lambda_{0} \sum_{l=1}^{m} \lambda_{l}+\sum_{i<j} \lambda_{i} \lambda_{j}\right]+\cdots
\end{array}
$$

Let us compare the coefficients of $\lambda^{m+1}$ in (20), (21), and the coefficients of $\lambda^{m}$ in (20), (21). We have

$$
\begin{gathered}
2 \lambda_{0}+\sum_{l=1}^{m} \lambda_{l}=\frac{1}{p}+\sum_{l=1}^{m} b_{l}, \\
\lambda_{0}^{2}+2 \lambda_{0} \sum_{l=1}^{m} \lambda_{l}+\sum_{i<j} \lambda_{i} \lambda_{j}=\frac{q}{p}+\frac{1}{p} \sum_{l=1}^{m} b_{l}+\sum_{i<j} b_{i} b_{j} .
\end{gathered}
$$


From (22), it follows that $2 \lambda_{0}=p^{-1}+\sum_{l=1}^{m}\left(b_{l}-\lambda_{l}\right)>p^{-1} \geqslant\left\|A^{-1 / 2}\right\|^{-2}$. Hence $\lambda_{0}>\left[2\left\|A^{-1 / 2}\right\|^{2}\right]^{-1}=\gamma_{1}$.

In what follows, we use the ideas from [6, p. 378]. Define $\delta:=2^{-1} \sum_{l=1}^{m}\left(b_{l}-\right.$ $\left.\lambda_{l}\right), \omega:=(2 p)^{-1}$. Then $\lambda_{0}=\omega+\delta($ see $(22))$. Extract $\sum_{l=1}^{m} \lambda_{l}$ from (22) and use this expression in (23). It follows that

$$
2 \delta \sum_{l=1}^{m} \lambda_{l}-\sum_{i<j}\left(b_{i} b_{j}-\lambda_{i} \lambda_{j}\right)=-\omega^{2}+2 \omega(\delta+q)-\delta^{2} .
$$

From $\lambda_{l} \in\left(b_{l-1}, b_{l}\right), l=\overline{1, m}\left(b_{0}:=0\right)$, one can obtain the following inequality (see [6, p. 380, Formula (5.24)]):

$$
\sum_{i<j}\left(b_{i} b_{j}-\lambda_{i} \lambda_{j}\right)<\sum_{j=1}^{m}\left(b_{j}-\lambda_{j}\right)\left(\sum_{i=1}^{m} \lambda_{i}\right)=2 \delta \sum_{l=1}^{m} \lambda_{l} .
$$

From (25), it follows that the right-hand part in (24) is positive. Consequently, $\omega<\delta+q+\left(2 \delta q+q^{2}\right)^{1 / 2}$. Therefore, $\lambda_{0}<2 \delta+q+\left(2 \delta q+q^{2}\right)^{1 / 2} \leqslant b_{m}+\|Q\|^{2}+$ $\|Q\|\left[b_{m}+\|Q\|^{2}\right]^{1 / 2}=\gamma_{2}$. Hence, $\lambda_{0} \in\left(\gamma_{1}, \gamma_{2}\right)$.

Theorem 2 implies $0 \notin \sigma_{p}(\mathscr{A})$. Hence, $0 \notin s(\mathscr{A})$.

Let $b_{q} \notin\left(\gamma_{1}, \gamma_{2}\right)$. Then from inequalities $b_{q} \leqslant b_{m}<\gamma_{2}$ we obtain $b_{q} \leqslant \gamma_{1}$. Let us suppose that $b_{q} \leqslant \gamma_{1}$ and $\operatorname{Ker}\left(\mathscr{A}-b_{q}\right)$ is degenerated. This means that there exists $\xi_{0} \in \operatorname{Ker}\left(\mathscr{A}-b_{q}\right)$ such that $\left[\xi_{0}, \xi\right]=0$ for all $\xi \in \operatorname{Ker}\left(\mathscr{A}-b_{q}\right)$. In particular, $\left[\xi_{0}, \xi_{0}\right]=0$.

Let $\xi_{0} \in \operatorname{Ker}\left(\mathscr{A}-b_{q}\right)$. Then (10) implies

$$
\begin{cases}A^{1 / 2}\left[A^{1 / 2} \vec{u}_{0}-\varphi_{0}^{1 / 2} Q^{*} r_{0}-\sum_{l=1}^{m}\left[\frac{\rho_{0} k_{l}}{b_{l}}\right]^{1 / 2} Q^{*} r_{l 0}\right]-b_{q} \vec{u}_{0}=0, \\ \varphi_{0}^{1 / 2} Q A^{1 / 2} \vec{u}_{0}-b_{q} r_{0} & =0, \\ {\left[\frac{\rho_{0} k_{l}}{b_{l}}\right]^{1 / 2} Q A^{1 / 2} \vec{u}_{0}+\left(b_{l}-b_{q}\right) r_{l 0}} & =0, l=\overline{1, m}, l \neq q, \\ {\left[\frac{\rho_{0} k_{q}}{b_{q}}\right]^{1 / 2} Q A^{1 / 2} \vec{u}_{0}} & =0 .\end{cases}
$$

Multiplying the first equation by $\vec{u}_{0}$ and using the other equations, we rearrange it to the form $\left\|\vec{v}_{0}\right\|_{H}^{2}=b_{q}\left\|A^{-1 / 2} \vec{v}_{0}\right\|_{H}^{2}$, where $\vec{v}_{0}:=A^{1 / 2} \vec{u}_{0}$. Hence $b_{q} \geqslant$ $\left\|A^{-1 / 2}\right\|^{-2}>\gamma_{1}$, which contradicts $b_{q} \leqslant \gamma_{1}$.

From the above, it follows that $s(\mathscr{A}) \subset\left(\gamma_{1}, \gamma_{2}\right)$, and the second statement of the theorem is proven.

3. The first parts of the statements 3 and 4 follow directly from [5, p. 271, Theorem 2.12]. If $\gamma_{2} \leqslant \gamma_{1}$, then $s(\mathscr{A})=\emptyset$ and the operator $\mathscr{A}$ has no non-real 
eigenvalues. Consequently, $\mathfrak{F}_{0}(\mathscr{A})=\mathscr{H}$. Hence the eigenelements of the operator $\mathscr{A}$ form a $\mathscr{G}$-orthonormal basis in $\mathscr{H}$.

3.2. Representation of solution of evolution equation. Let us assume that $\gamma_{2} \leqslant \gamma_{1}$. Then from Theorem 5 it follows that the eigenelements of the operator $\mathscr{A}$ form a $\mathscr{E}$-orthonormal basis in $\mathscr{H}$. This basis is also a $p$-basis $(p>3)$ in $\mathscr{H}$. By Theorem 3, this basis (after being divided into positive and negative elements) can be represented in the form

$$
\begin{gathered}
\left\{\xi_{k}^{ \pm}:=\left(A^{-1 / 2} \vec{v}_{k}^{ \pm} ;\left(\mathscr{G}-\lambda_{k}^{ \pm}\right)^{-1} Q \vec{v}_{k}^{ \pm}\right)^{\tau}\right\}_{k=1}^{\infty}, \\
\xi_{k}^{ \pm} \in L_{ \pm}\left(\mathcal{A}^{-1}\right), \quad\left[\xi_{k}^{+}, \xi_{j}^{+}\right]=\delta_{k j}, \quad\left[\xi_{k}^{-}, \xi_{j}^{-}\right]=-\delta_{k j}, \quad\left[\xi_{k}^{+}, \xi_{j}^{-}\right]=0 .
\end{gathered}
$$

Let us represent the solution $\xi(t)$ of problem (7) in the following form:

$$
\xi(t)=\sum_{k=1}^{\infty} c_{k}^{+}(t) \xi_{k}^{+}+\sum_{j=1}^{\infty} c_{j}^{-}(t) \xi_{j}^{-}, \quad c_{k}^{+}(0)=\left[\xi^{0}, \xi_{k}^{+}\right], \quad c_{j}^{-}(0)=-\left[\xi^{0}, \xi_{j}^{-}\right] .
$$

From $(7),(26),(27)$, the formulae for $\xi^{0}$ and $\mathscr{F}(t)$, we find that

$$
\begin{gathered}
\xi(t)=\sum_{k=1}^{\infty}\left(e^{-\lambda_{k}^{+} t}\left[\xi^{0}, \xi_{k}^{+}\right]+\int_{0}^{t} e^{-\lambda_{k}^{+}(t-s)}\left[\mathscr{F}(s), \xi_{k}^{+}\right] d s\right) \xi_{k}^{+} \\
\quad-\sum_{j=1}^{\infty}\left(e^{-\lambda_{j}^{-} t}\left[\xi^{0}, \xi_{j}^{-}\right]+\int_{0}^{t} e^{-\lambda_{j}^{-}(t-s)}\left[\mathscr{F}(s), \xi_{j}^{-}\right] d s\right) \xi_{j}^{-}, \\
{\left[\xi^{0}, \xi_{k}^{ \pm}\right]=\left(\vec{u}^{0}, A^{-1 / 2} \vec{v}_{k}^{ \pm}\right)_{H}-\frac{\varphi_{0}}{\lambda_{k}^{ \pm}}\left(\rho^{0}, Q \vec{v}_{k}^{ \pm}\right)_{L_{2, \Omega}}, \quad k \in \mathbb{N},} \\
{\left[\mathscr{F}(t), \xi_{k}^{ \pm}\right]=\left(\vec{f}(t), A^{-1 / 2} \vec{v}_{k}^{ \pm}\right)_{H}+\sum_{l=1}^{m} \frac{\rho_{0} k_{l} e^{-b_{l} t}}{b_{l}}\left(\rho^{0}, Q \vec{v}_{k}^{ \pm}\right)_{L_{2, \Omega}}, \quad k \in \mathbb{N} .}
\end{gathered}
$$

From (28), we obtain the representation of the solution $\vec{u}(t), \rho(t)$ of problem (2) with respect to a system of eigenvectors $\left\{\vec{v}_{k}^{ \pm}\right\}_{k=1}^{+\infty}$ of the operator pencil $L(\lambda)$ (this system is connected with $\mathscr{g}$-orthonormal basis (26) and is normalized in a special way):

$$
\begin{aligned}
&\left(\begin{array}{c}
\vec{u}(t) \\
\rho(t)
\end{array}\right)= \sum_{k=1}^{\infty}\left[T_{k}^{+}(t)\left(\begin{array}{c}
A^{-1 / 2} \vec{v}_{k}^{+} \\
\left(\lambda_{k}^{+}\right)^{-1} Q \vec{v}_{k}^{+}
\end{array}\right)-T_{k}^{-}(t)\left(\begin{array}{c}
A^{-1 / 2} \vec{v}_{k}^{-} \\
\left(\lambda_{k}^{-}\right)^{-1} Q \vec{v}_{k}^{-}
\end{array}\right)\right] \\
& T_{k}^{ \pm}(t):=\int_{0}^{t} e^{-\lambda_{k}^{ \pm}(t-s)}\left(\vec{f}(s), A^{-1 / 2} \vec{v}_{k}^{ \pm}\right)_{H} d s+e^{-\lambda_{k}^{ \pm} t}\left(\vec{u}^{0}, A^{-1 / 2} \vec{v}_{k}^{ \pm}\right)_{H} \\
&+\left[\sum_{l=1}^{m} \frac{\rho_{0} k_{l}\left(\lambda_{k}^{ \pm} e^{-b_{l} t}-b_{l} e^{-\lambda_{k}^{ \pm} t}\right)}{\lambda_{k}^{ \pm} b_{l}\left(\lambda_{k}^{ \pm}-b_{l}\right)}-\frac{e^{-\lambda_{k}^{ \pm} t}}{\lambda_{k}^{ \pm}}\right]\left(\rho^{0}, Q \vec{v}_{k}^{ \pm}\right)_{L_{2, \Omega}} .
\end{aligned}
$$

The author thanks Professor N.D. Kopachevsky for attention to this work and the anonymous referee for useful suggestions. 
On Properties of Root Elements ... Small Motions of Viscous Relaxing Fluid

\section{References}

[1] D. Zakora, On the Spectrum of Rotating Viscous Relaxing Fluid, Zh. Mat. Fiz. Anal. Geom. 12 (2016), No. 4, 338-358.

[2] D. Zakora, A Symmetric Model of Viscous Relaxing Fluid. An Evolution Problem, Zh. Mat. Fiz. Anal. Geom. 8 (2012), No. 2, 190-206.

[3] A.S. Marcus, Introduction to Spectral Theory of Polinomial Operator Pencils, Shtiinca, Kishenev, 1986 (Russian).

[4] D.A. Zakora, Operator Approach to Ilushin's Model of Viscoelastic Body of Parabolic Type, Sovrem. Mat. Fundam. Napravl. 57 (2015), 31-64 (Russian); Engl. transl.: J. Math. Sci. (N.Y.) 225 (2015), No. 2, 345-380.

[5] T.Ya. Azizov and I.S. Iohvidov, Basic Operator Theory in Spaces with Indefinite Metrics, Nauka, Moscow, 1986 (Russian).

[6] N.D. Kopachevsky and S.G. Krein, Operator Approach to Linear Problems of Hydrodynamics. Vol. 2: Nonself-Adjoint Problems for Viscous Fluids, Birkhäuser Verlag, Basel-Boston-Berlin, 2003. 